\title{
Star formation in the solar neighbourhood
}

\author{
Juvela, Mika
}

Cambridge University Press

2020

Juvela , M 2020 , Star formation in the solar neighbourhood . in B G Elmegreen , L V Tóth \&

$M$ Güdel (eds), Origins : From the Protosun to the First Steps of Life (IAU S345) .

Proceedings of the International Astronomical Union, no. S345, vol. 14 , Cambridge

University Press , Cambridge , pp. 15-22 , IAU Symposia , Vienna , Austria , 20/08/2018 . https://doi.org/10.1017/S1

http://hdl.handle.net/10138/328929

https://doi.org/10.1017/S1743921319001911

acceptedVersion

Downloaded from Helda, University of Helsinki institutional repository.

This is an electronic reprint of the original article.

This reprint may differ from the original in pagination and typographic detail.

Please cite the original version. 


\title{
Star formation in the solar neighbourhood
}

\author{
Mika Juvela \\ Department of physics, University of Helsinki, P.O. Box 64 (Gustaf Hällströmin katu 2), \\ FI-00014 University of Helsinki, Finland \\ email: mika.juvela@helsinki.fi
}

\begin{abstract}
Star formation started as a cosmic process soon after the big bang and still continues in the Milky Way, although at a decreasing rate. The formation of dense interstellar clouds, their fragmentation and eventual collapse lead to the birth of stars. The nearby clouds provide the highest resolution for the study of this process. The progress is closely following the improvement of the infrared and radio-wavelength facilities that enables us to follow even the earliest stages of the star-formation process inside molecular clouds. On the other hand, modern numerical simulations can take into account most of the relevant physics and often provide a more direct access into the general principles of star formation. The comparison of observations and simulations is therefore essential. In this paper, will discuss star formation in the solar neighbourhood, concentrating on the prestellar phases leading up to the formation of protostars.
\end{abstract}

Keywords. stars: formation, ISM: clouds, ISM: kinematics and dynamics, ISM: structure, ISM: magnetic fields, dust, extinction, infrared: ISM, radio lines: ISM, submillimeter

\section{Nearby molecular clouds}

There are many nearby molecular clouds at distances just beyond 100 pc (see Fig. 1). Because most instruments, for example single-dish radio telescopes, provide an angular resolution better than $\sim 1$ arcmin, star formation in these clouds can be studied from clouds scales (sizes $>1 \mathrm{pc}$ ) to clumps $(\sim 1 \mathrm{pc})$ and down to the structure of gravitationally bound cores $(\sim 0.1 \mathrm{pc})$ (Bergin \& Tafalla 2007). The nearby clouds have a central role when we try to understand the formation of low and intermediate mass stars.

Many of the best-studied clouds belong to the Gould Belt. This structure is tilted by some $20^{\circ}$ with respect to the Galactic plane and was first recognised in the distribution of bright stars in the southern sky. B.A. Gould referred to it already in 1874 (Gould 1874) and wrote in his Uranometria Argentina: "Thus I cannot avoid the conviction that our own system forms part of a small cluster, distinct from the vast organization of that forms the Milky Way..." (Gould 1879). This is not very far from the current understanding. The belt is not only a stellar structure but also a location of on-going star formation, containing regions such as the Taurus-Perseus-Auriga complex (distances $d \sim 100-500 \mathrm{pc})$, the $\rho$ Ophiuchus cloud $(d \sim 150 \mathrm{pc})$, and the Orion region $(d \sim 400 \mathrm{pc})$.

Recent observations have provided a wealth of new information on the nearby clouds. Even now, the data are still revealing entirely new phenomena that are related to the structure of the clouds and the final stages of the prestellar evolution.

\section{Observations of nearby clouds}

Ground-based observations of molecular lines and dust continuum extend from radio wavelengths (Myers et al. 1983, Motte et al. 1998) to sub-millimetre (Ward-Thompson et al. 2000, Shirley et al. 2000). The past two decades have seen a number of spaceborne instruments that have complemented these data with infrared and sub-millimetre 
observations of unprecedented sensitivity. These include satellites such as ISO (Kessler et al. 1996; e.g. Tóth et al. 2000), Spitzer (Werner et al. 2004), AKARI (Murakami et al. 2007), and WISE (Wright et al. 2010). The combination of different instruments makes it possible to follow the full evolution from cold prestellar clouds to protostellar cores, before the young stellar objects (YSOs) again come within the reach of groundbased near-infrared telescopes. Together the ground-based and the space-borne infrared instruments have also made it possible to catalogue YSOs in the nearby clouds and even over the whole sky.

The Herschel Space Observatory (Pilbratt et al. 2010) has made a particularly large impact on star-formation studies. It mapped the whole Galactic plane (Molinari et al. 2010) and most nearby molecular clouds (André et al. 2010) and regions of high-mass star formation (Motte et al. 2010). The multi-frequency sub-millimetre continuum data literally shed new light on the star-formation problem by showing the intricate, extended density structures around the star-forming cores. This is in contrast with ground-based continuum surveys where the angular resolution is usually higher but the imaging of extended emission is more challenging (Schuller et al. 2009, Ward-Thompson et al. 2016). Herschel also carried out heterodyne observations of many key lines that are inaccessible from the ground (e.g. Mottram et al. 2014, Goicoechea et al. 2015).

One should also not forget extinction measurements from optical to mid-infrared wavelengths (Cambrésy 1999, Bacmann et al. 2000, Alves et al. 2001). These provide an important point of comparison by being independent of dust temperature. Near-infrared extinction also appears to be little affected by dust evolution compared to the variations seen in the long-wavelength dust opacity (Cardelli et al. 1989, Wang \& Jiang 2014). With ground-based surveys one can cover everything from diffuse regions $\left(A_{\mathrm{V}}<1 \mathrm{mag}\right)$ to cores with tens of magnitudes in $A_{\mathrm{V}}$ (Kandori et al. 2005, Juvela et al. 2008). Nearby clouds have thus been studied combining emission and extinction (Cambrésy et al. 2001, Lombardi et al. 2006, 2014, Meingast et al. 2018) and even all-sky extinction maps exist The ambiguity resulting from the line-of-sight (LOS) mixing of the stellar and interstellar matter distributions is being addressed with 3D extinction mapping methods (Marshall et al. 2009, Green et al. 2018, Lallement et al. 2018).

While space instruments provide improved sensitivity and wavelength coverage, groundbased telescopes still excel in resolution. Radio interferometers, especially ALMA, are

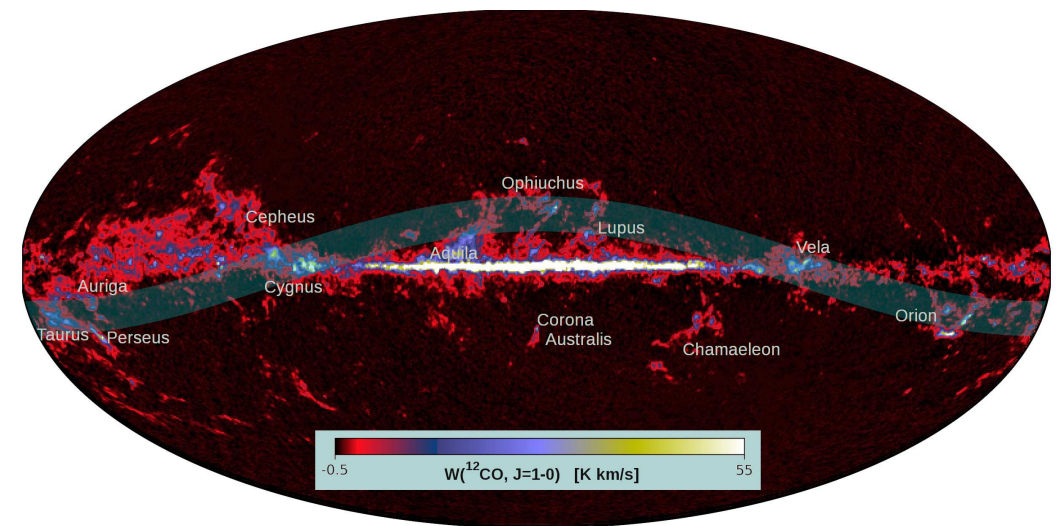

Figure 1. Some nearby molecular clouds marked on the all-sky CO line emission map derived from Planck measurements (Planck Collaboration 2014). These clouds are important targets of star-formation studies, thanks to their typically small distances $(100-500 \mathrm{pc})$. Many of the clouds belong to the Gould Belt system (Gould 1879) that is indicated with a cyan band. 
revealing new details in the density structure, kinematics, and chemistry of star-forming clouds at the very smallest scales (Kainulainen et al. 2017, Hacar et al. 2018) and especially at the protostellar phases (e.g. Cox et al. 2018).

\section{Structure of star-forming clouds}

Herschel made cloud filaments a central topic of star-formation studies. Naked-eye observations of dark nebulae go back to William Herschel (Herschel 1785) but the first observations of elongated cloud structures came with astrophotography. E. E. Barnard commented on the dark lanes, streaks, and thin threads visible in his photographs (Barnard 1910). Already in 1894, A.C. Ranyard described the $\rho$ Ophiuchus images as showing "dark vacant areas or channels... seem to me to be undoubtedly dark structures, or obscuring masses in space, which cut out the light from the nebulous or stellar region behind them". Barnard cautiously agreed that these were caused by matter in the interstellar space. The appearance of Pleiades and the Orion nebula was suggestive of a connection between the filamentary nebulosities and stars. Theoretical studies of filament fragmentation and star formation in this context go back at least 70 years (Edgeworth 1946).

Although filaments were later seen in simulations of hydrodynamical and magnetohydrodynamical turbulence (e.g. Padoan et al. 2001) they were less evident in observations and only rarely a central topic in star-formation studies (Elmegreen \& Elmegreen 1979, Bally et al. 1987, Enoch et al. 2007). This changed fundamentally with Herschel (the satellite). By imaging large areas with a sensitivity far exceeding that of ground-based instruments (down to below $N\left(\mathrm{H}_{2}\right)=10^{21} \mathrm{~cm}^{-2}$ ), it showed the extensive filamentary networks in both diffuse clouds and actively star-forming regions (André et al. 2010, Miville-Deschênes et al. 2010, Arzoumanian et al. 2011).

The radial profiles of the filaments are observed to be flat compared to the $\rho \sim r^{-4}$ behaviour predicted for isothermal cylinders in hydrostatic equilibrium (Stodólkiewicz 1963, Ostriker 1964). This suggests effects of temperature variations or magnetic fields - or that filaments indeed are not static structures (Kawachi \& Hanawa 1998, Nakamura \& Umemura 1999, Fiege \& Pudritz 2000, Palmeirim et al. 2013, Arzoumanian et

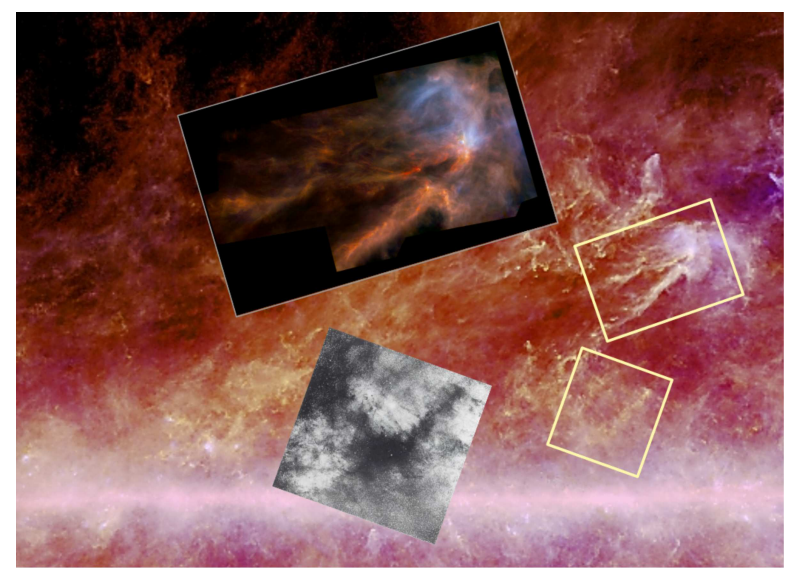

Figure 2. Filamentary clouds in the Ophiuchus region. The background shows surface brightness measurements from Planck (Planck Collaboration 2016a) combined with IRAS $100 \mu \mathrm{m}$ data (credits: ESA/HFI Consortium/IRAS). The zoom-ins show the main $\rho$ Oph cloud (upper box and inset) as seen with Herschel (credit: ESA/Herschel/NASA/JPL-Caltech, R. Hurt 2017), and the Pipe nebula photographed by E.E. Barnard some 110 years earlier (Barnard 1926). 
al. 2013). The filaments of nearby clouds also appear to have a narrow distribution of widths, $\sim 0.05-0.2 \mathrm{pc}$ (Arzoumanian et al. 2011, Rivera-Ingraham et al. 2016). However, in images one can readily see structures at different scales (Fig. 2). Thick filaments may consist of sub-filaments and the width can vary along the filaments, possibly correlated with the column density and core formation (Juvela et al. 2012a, Heigl et al. 2018). Width measurements are complicated by the varying background, radiative transfer effects, and the finite resolution of the observations (Juvela et al. 2012b, Panopoulou 2017, Ossenkopf-Okada \& Stepanov 2018). Nevertheless, the observations have lead to attempts to theoretically explain the preferred filament widths (e.g. Fischera \& Martin 2012, Hennebelle \& André 2013, André et al. 2014, Xu \& Lazarian 2018).

Molecular line observations revealed in some filaments velocity-coherent substructures that were coined fibers (Hacar \& Tafalla 2011). Without full 3D information, detections in the position-position-velocity (PPV) space might suffer from LOS confusion and a PPV structure might not necessarily be identifiable in 3D density (Clarke et al. 2018). However, interferometric observations have detected fibers of high column density, narrow width, and almost subsonic velocity dispersion. This suggests that the fibers represent another step closer to the actual star formation (Hacar et al. 2018, Monsch et al. 2018).

Low-column-density striations are observed perpendicular to the major axis of some more massive structures (Peretto et al. 2013, Cox et al. 2016). These can be seen also in line emission, for example in Taurus (Heyer al. 2016). The correlation with polarisation suggests a close connection to the magnetic fields. Observations and simulations show that massive filaments tend to be perpendicular to the magnetic field while low-density structures are preferably parallel (Planck Collaboration et al. 2016b, Malinen et al. 2016 Soler \& Hennebelle 2017). Striations could thus represent accretion onto filaments. This is seen in some simulations but is difficult to quantify in observations because of the unknown inclination (Vestuto et al. 2003, Palmeirim et al. 2013, Chen et al. 2017).

The projected shape of clouds is known accurately from observations but, in the LOS direction, we are only now starting to get constraints from 3D extinction mapping and from YSO and field star distance measurements (Green et al. 2018, Galli et al. 2018, Großschedl et al. 2018). The situation could be better for cloud sub-structures if one could assume that these follow some symmetries. Cores and clumps are often modelled with radial density distributions (Shirley et al. 2000, Juvela et al. 2018). These may be adequate for quasi-static cores but, because clumps do have sub-structure, spherical models only provide a statistical characterisation of these objects.

Similarly, Herschel filaments are known to have internal structure that is not detected in low-resolution data or without the benefit of velocity information. One may also ask whether they are more cylindrically symmetric objects or sometimes only tangential projections of two-dimensional sheets. The large contrast between the (star-forming) filaments and the background and the quasi-uniform width would seem to argue against the sheet hypothesis. If the structures were extended in the LOS direction, we would also not see such a clear connection between the filaments and the striations. More directly, measurements of high volume densities can show that the mass seen in column density is concentrated in a small volume (Shimajiri et al. 2018). Objects such as the Musca cloud contain, or partly even consists of, clumps that have a structure reminiscent of Bonnor-Ebert spheres (Juvela et al. 2010). If the filaments were only 2D projections, the alignment between clumps and the filaments would be worse. The same argument applies to the tight spatial correlation with the prestellar and protostellar cores. Filaments may reside in sheets or be the product of sheet collisions but at least the dense ones appear to be closer to the cylinder symmetry. However, the cylindrical shape is only a very crude approximation of the complex structure of the real filaments. 


\section{Birth of prestellar cores}

Cloud fragmentation leads to the formation prestellar and protostellar cores (see the review by $\mathrm{C}$. Federrath in this issue). These are found in regions with background column densities above $N\left(\mathrm{H}_{2}\right) \sim 5 \times 10^{21} \mathrm{~cm}^{-2}\left(\Sigma \sim 110 \mathrm{M}_{\odot} \mathrm{pc}^{-2}\right)$ (André et al. 2010, Lada et al. 2010, Montillaud et al. 2015, Marsh et al. 2016). Since star formation progresses from atomic clouds to stellar densities, it would seem that almost any "threshold" could be selected by concentrating on a different size scale. However, the coincidence with the threshold of filament stability suggests that this limit is of a more fundamental nature.

The spatial distribution of protostellar sources is strongly associated to super-critical filaments (Könyves et al. 2015, Marsh et al. 2016, Rivera-Ingraham et al. 2017, Bresnahan et al. 2018). Isothermal filaments should fragment when the mass per length exceeds the critical value, $M_{\text {crit }}=2 c_{\mathrm{s}}^{2} / G$, but one should take into account also the effects of internal non-thermal motions, external pressure, magnetic fields, the non-uniform turbulent environment and other external agents (see Fig. 3) - and the dynamical nature of the filaments themselves (Inutsuka \& Miyama 1997, Fiege \& Pudritz 2000, Myers et al. 2009, Heitsch et al. 2011, Seifried \& Walch 2015, Haugbølle et al. 2018).

Cloud and filament properties show systematic trends but each cloud is individual and during its lifetime may be subjected to stellar winds and repeated supernova shocks (Peretto et al. 2012, Inutsuka et al. 2015). The conditions set by random density and velocity fluctuations affect the structure and the fragmentation. Although, e.g. the observed fragmentation lengths are often attributed to some gravitational or MHD instability, it is not surprising that real clouds do not strictly follow the theoretical predictions (Beuther et al. 2015, Roy et al. 2015, Contreras et al. 2016, Kainulainen et al. 2017).

Star formation is characterised by the star-formation efficiency (SFE) and the stellar initial mass function (IMF). To explain the origin of these, one must characterise the preceding step, the prestellar cores, looking at the corresponding core formation efficiency (CFE, the fraction of cloud mass converted into cores) and core mass function (CMF) (Motte et al. 1998, Kiss et al. 2006, Alves et al. 2007). CFE estimates vary in wide range (at least $5-35 \%$ ) between different clouds, probably also depending on the observations used and the chosen extent of the region (Palau et al. 2013, Zhang et al. 2018, Kong et al. 2018). Many studies have commented on the similarity of the IMF and the prestellar CMF after a $\sim 30 \%$ relative shift (efficiency) in masses (e.g. Marsh et al. 2016, Sokol et al. 2018). Compared to the local clouds, CMF seems to be flatter in high-pressure environments and regions of high-mass star formation (Liu et al. 2018). However, it is still unclear how sensitive (or insensitive) the CMF is to the the local cloud conditions.
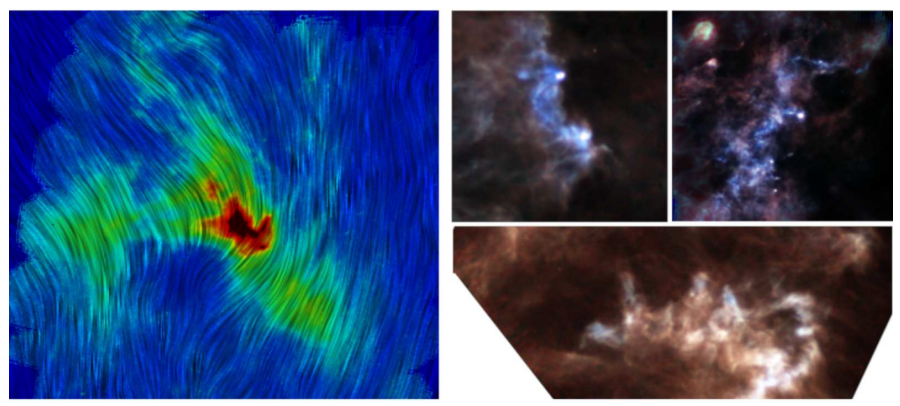

Figure 3. Left: Herschel intensity map of the star-forming cloud LDN 1642. The line integral convolution (LIC) pattern shows the plane-of-the-sky magnetic field orientation based on the $353 \mathrm{GHz}$ Planck data (Malinen et al. 2016). Right: Herschel images of three star-forming clouds where the filamentary structure is strongly modified by external forces (Juvela et al. 2012). 


\section{Outlook}

Many open question remain concerning star formation in the solar neighbourhood. How are the filaments formed, how do they acquire their final mass, what governs their fragmentation to cores, and what are the lifetimes of all the objects? This should depend on whether evolution is driven mainly by turbulence or gravity, whether external forces (radiation, stellar winds, and supernovae) are important, and what role the still poorly known magnetic fields have (Fig. 3). This complexity makes it difficult to determine the environmental differences that would explain cloud-to-cloud variations of the SFE and the IMF, to the extent they indeed exist. It also limits the generality of the conclusions that can be drawn from any limited cloud sample. This may apply even to the entire sample of nearby clouds, where the influence of massive stars is limited.

Unlike simulations, observations cannot be used to follow the formation of a star from start to finish. We only have a series of snapshots of different environments in different stages of evolution. This has not prevented the determination of many generalities, from the statistics of the large-scale density field (e.g. Kainulainen et al. 2009) to the CMF properties. Progress clearly needs close collaboration between observers and modellers. We should also reach a point where, instead of only comparing observations to random model realisations, accurate models are developed for the real individual clouds.

\section{References}

Alves, J. F., Lada, C. J., Lada, E. A. 2001, Nature 409, 159

Alves, J., Lombardi, M., Lada, C. J. 2007, A\&A, 462, L17

André, Ph., Men'shchikov, A., Bontemps, S., et al. 2010, A\& A, 518, L102

André, P., Di Francesco, J., Ward-Thompson, D., et al. 2014, in: H. Beuther, R. S. Klessen, C. P. Dullemond, \& T., Henning (eds.), Protostars and Planets VI, University of Arizona Press, Tucson, p. 27

Arzoumanian, D., André, Ph., Didelon, P., Könyves, V., Schneider, et al. 2011, A\&A 529, L6

Arzoumanian, D., Shimajiri, Y., Inutsuka, S., Inoue, T., \& Tachihara, K. 2018, PASJ 70, 96

Bacmann, A., André, P., Puget, J.-L., et al. 2000, A\&A 361, 555

Bally, J., Langer, W. D., Stark, A. A., Wilson, R. W. 1987, ApJ (Letters), 312, L45

Barnard, E. E. 1910, ApJ 31, 8

Barnard, E. E., A Photographic Atlas of Selected Regions of the Milky Way. Ed. Edwin B. Frost and Mary R. Calvert. Carnegie Institution of Washington, 1927

Beichman, C. A., Neugebauer, G., Habing, H. J., et al. 1988, Infrared Astronomical Satellite (IRAS) Catalogs and Atlases, Vol. 1: Explanatory Supplement (Washington, DC: GPO)

Bergin, E. A., Tafalla, M. 2007, ARAA 45, 339

Beuther, H., Ragan, S. E., Johnston, K., et al. 2015, $A \& A$, 584, A67

Bresnahan, D., Ward-Thompson, D., Kirk, et al. 2018, A\&A 615, A125

Cambrésy, L. 1999, A\&A 345, 965

Cambrésy, L., Boulanger, F., Lagache, G., \& Stepnik, B. 2001, A\&̊A, 375, 999

Cardelli, J. A., Clayton, G. C., \& Mathis, J. S. 1989, ApJ 345, 245

Caselli, P., Keto, E., Bergin, E. A., et al. 2012, ApJ (Letters), 759, L37

Clarke, S. D., Whitworth, A. P., Spowage, R. L., et al. 2018, MNRAS, 479, 1722

Contreras, Y., Garay, G., Rathborne, J. M., Sanhueza, P. 2016, MNRAS, 456, 2041

Cox, N. L. J., Arzoumanian, D., André, Ph., et al. 2016, A\&A 590, A110

Cox, E. G., Harris, R. J., Looney, et al. 2018, ApJ 855, 92

Elmegreen, D. M., Elmegreen, B. G. 1979, AJ 84, 5

Enoch, M. L., Glenn, J., Evans, N. J., II, Sargent, A. I., Young, K. E., Huard, T. L. 2007, ApJ, 666,982

Fiege, J. D., \& Pudritz, R. E. 2000, MNRAS, 311, 85

Galli, P. A. B., Loinard, L., Ortiz-Léon, G. N., et al. 2018, ApJ, 859, 33

Goicoechea, J. R., Teyssier, D., Etxaluze, M., et al. 2015, ApJ 812, 75 
Gould, B.A. 1879, Resultados del Observatorio Nacional Argentino, vol. 1, 1

Gould, B.A. 1874, The American Journal of Science and Arts (Third Series), Vol. 8, 325

Green, G. M., Schlafly, E. F., Finkbeiner, D., et al. 2018, MNRAS 478, 651

Großschedl, J. E., Alves, J., Meingast, S., et al. 2018, A\& $A$, 619, A106

Hacar, A., \& Tafalla, M. 2011, A\& $A, 533,34$

Hacar, A., Tafalla, M., Forbrich, J., et al. 2018, A\&A, 610, A77

Haugb $\varnothing l l e$, T., Padoan, P., \& Nordlund, §̊ 2018, ApJ, 854, 35

Heigl, S., Gritschneder, M., Burkert, A. 2018, MNRAS 2018, 481, L1

Heitsch, F., Naab, T., Walch, S. 2011, MNRAS 415, 271

Hennebelle, P., \& André, Ph. 2013, A\&̈A 560, A68

Herschel, W., 1785, Philosophical Transactions, 75, 213

Inutsuka, S., Inoue, T., Iwasaki, K., Hosokawa, T. 2015, A\&A, 580, A49

Juvela, M., Pelkonen, V.-M., Padoan, P., Mattila, K. 2008, A\& A 480, 445

Juvela, M., Ristorcelli, I., Montier, L. A., et al. 2010, $A \mathscr{E} A$, 518, L93

Juvela, M., Ristorcelli, I., Pagani, L., et al. 2012a, $A \mathscr{G} A$ 541, A12

Juvela, M., Malinen, J., Lunttila, T. 2012b, A\&A 544, A141

Juvela, M., Malinen, J., Montillaud, J., et al. 2018, A\&A, 614, A83

Kainulainen, J., Federrath, C., \& Henning, T. 2014, Science, 344, 183

Kainulainen, J., Stutz, A. M., Stanke, T., et al. 2017, A\&SA 600, A141

Kandori, R., Nakajima, Y., Tamura, M., et al. 2005, AJ 130, 2166

Kawachi, T. \& Hanawa, T. 1998, PASJ 50, 577

Kessler, M. F., Steinz, J. A., Anderegg, M. E., et al. 1996, A\&3A 315, L27

Kiss, Z. T., Tóth, L. V., Krause, O., et al. 2006, A\&A 453, 923

Kong, S., Tan, J. C., Arce, H. G., et al. 2018, ApJ (Letters), 855, L25

Könyves, V., André, Ph., Men'shchikov, A., et al. 2015, A\&\&A 584, A91

Krumholtz, M. 2014, Phys. Rep., 539, 49

Lallement, R., Capitanio, L., Ruiz-Dern, L., et al. 2018, A\& A 616, A132

Liu, M., Tan, J. C., Cheng, Y., Kong, S. 2018, ApJ 862, 105

Lombardi, M., Alves, J., Lada, C. J. 2006, A $\dot{E} A$ 454, 781

Lombardi, M., Bouy, H., Alves, J., Lada, C. J. 2014, A\&A 566, A45

Malinen, J., Montier, L., Montillaud, J., et al. 2016, MNRAS 460, 1934

Marsh, K. A., Kirk, J. M., André, Ph., et al. 2016, MNRAS 459, 342

Marshall, D. J., Joncas, G., Jones, A. P. 2009, ApJ 706, 727

Meingast, S., Alves, J., Lombardi, M. 2018, A\&A 614, A65

Miville-Deschênes, M.-A., Martin, P. G., Abergel, A., et al. 2010, A\&SA 518, L104

Molinari, S., Swinyard, B., Bally, J., et al. 2010, $A \& A A$ 518, L100

Monsch, K., Pineda, J. E., Liu, H. B., et al. 2018, ApJ 861, 77

Motte, F., André, P., Neri, R. 1998, A\&A 336, 150

Motte, F., Zavagno, A., Bontemps, S., et al. 2010, A\&A 518, L77

Mottram, J. C., Kristensen, L. E., van Dishoeck, E. F., et al. 2014, A\&A 572, A21

Murakami, H. Baba, H., Barthel, P., et al. 2007, PASJ 59, 369

Myers, P. C., Linke, R. A., Benson, P. J. 1983, ApJ 264, 517

Myers, P. C. 2009, ApJ 700, 1609

Nakamura, F., \& Umemura, M. 1999, ApJ 515, 239

Ossenkopf-Okada, V., \& Stepanov, R. 2018, A\& $A$ (in press), arxiv:1811.02082

Ostriker, J. 1964, ApJ 140, 1056

Padoan, P., Juvela, M., Goodman, A. A., Nordlund, Åke 2001, ApJ 553, 227

Palau, A., Fuente, A., Girart, J. M., et al. 2013, ApJ, 762, 120

Palmeirim, P., André, Ph., Kirk, J., et al. 2013, A\&A 550, A38

Panopoulou, G. V., Psaradaki, I., Skalidis, R., et al. 2017, MNRAS 466, 2529

Pilbratt, G. L., Riedinger, J. R., Passvogel, T., et al. 2010, A\&A 518, L1

Planck Collaboration, 2014, A\&SA 571, A13

Planck Collaboration, 2016a, $A \mathscr{E} A$ 594, A1

Planck Collaboration 2016b, A\&\&A 586, A135 
Rivera-Ingraham, A., Ristorcelli, I., Juvela, M., et al. 2017, A\&A 601, A94

Roy, A., André, Ph., Arzoumanian, D., et al. 2015, A\&A, 584, A111

Seifried, D., \& Walch, S. 2015, MNRAS, 452, 2410

Shirley, Y. L., Evans, N. J., II, Rawlings, J. M. C., Gregersen, E. M. 2000, ApJS 131, 249

Sokol, A. D., Gutermuth, R. A., Pokhrel, R., et al. 2019, MNRAS 483, 407

Soler, J. D., \& Hennebelle, P. 2017, A\&A 607, A2

Stodólkiewicz, J. S. 1963, Acta Astron. 13, 30

Tóth, L. V., Hotzel, S., Krause, O., et al. 2000, A\& $A$ 364, 769

Vestuto, J. G., Ostriker, E. C., \& Stone, J. M. 2003, ApJ, 590, 858

Wang, S., Jiang, B. W 2014, ApJ (Letters) 788, L12

Ward-Thompson, D., Kirk, J. M., Crutcher, R. M., et al. 2000, ApJ (Letters) 537, L135

Ward-Thompson, D., Pattle, K., Kirk, J. M., et al. 2016, MNRAS, 463, 1008

Werner, M., Roellig, T., Low, F., et al. 2004, ApJS, 154, 1

Wright, E. L., Eisenhardt, P. R. M., Mainzer, A. K., et al. 2010, AJ 140, 1868

Zhang, G.-Y., Xu, J.-L., Vasyunin, A. I., et al. 2018, $A \mathscr{E} A$, in press, arXiv:1810.09522

\section{Discussion}

Alfaro: Could you estimate the expected lifetime of filaments?

JUVELA: Filaments are fragmenting to cores and the lifetime of a core is known to be at least $\sim 1$ Myr. Clouds live at least a few million of years, so the lifetime of a filament must be in the same ball park

KhaIBRAKHMAnov: Filaments are cylinders or sheets? Can we distinguish between these structures?

JuvELA: Regarding cylindrical shape, filaments are likely cylinders. But it is not easy to determine their shape from observations. For some filaments, the volume density has been estimated from molecular lines, which suggest they are cylinders more than sheets.

ZINNECKER: Herschel observations put the filamentary structure of the dense interstellar medium into the limelight. However, in the historical context, who is to be credited with the filamentary nature of dark clouds?

JUVELA: Some credit should certainly be given to Barnard, who drew attention to these elongated structures within the ISM - even if he may not have used the filament word to describe them.

Tóтн: Filaments are usually seen as 2D objects. How much we can trust these are real objects and not just projections?

JUVELA: Simulations have shown that projection effects play a role and especially the filaments seen in continuum are not necessarily single objects in $3 \mathrm{D}$. They may be the superposition of several structures that are not physically connected. However, in the case of such filaments like Musca, it is very likely to be a single object, a real filament.

MAROV: Did you find any correlation between line slopes and fiber/filament structure?

JUVELA: Line profiles can be used to search for signs of collapse - but in the case of fibers, it is just their velocity, they being velocity-coherent in the ppv space.

HACAR: The fibers have narrow lines with clearly subsonic linewidths. 